\title{
Los Comités Asistenciales de Ética (CAE). Un año de funcionamiento del CAE del Área de Salud de Albacete
}

Marina Gascón Abellán ${ }^{a}$

\author{
${ }^{a}$ Miembro del Comité de Ética \\ Asistencial del Área de Salud \\ de Albacete. Catedrática de \\ Filosofía del Derecho De \\ la Facultad de Derecho de \\ Albacete. \\ Correspondencia: Marina \\ Gascón Abellán. E-mail: \\ marina.gascon@uclm.es.
}

Recibido el 12 de abril de 2010.

Aceptado para su publicación el 14 de mayo de 2010.

\begin{abstract}
RESUMEN
Los Comités de Ética Asistencial son órganos asesores cuya función principal (aunque no la única) consiste en aconsejar sobre el modo de actuar ante los problemas o controversias de carácter ético que puedan surgir en la práctica clínica. En los últimos años la presencia de estos comités en España ha experimentado un notable crecimiento. Al hilo de la normativa específica de Castilla-la Mancha, se repasan aquí las principales características de estos órganos (funciones, composición, modo de funcionamiento, etc.), y se concluye finalmente, a modo de balance, con una descripción somera del CEA de Albacete, tras algo más de un año desde su creación.
\end{abstract}

Palabras clave: Comités de Ética Clínica.

\section{ABSTRACT}

Healthcare Ethics Committees (HEC). One year of the HEC in the Albacete Health Region.

The main function (although not the only one) of Healthcare Ethics Committees is to give advice on how to act when faced with ethical problems or controversies in clinical practice. Over the last few years there has been a considerable growth of such entities in Spain. Based on the specific regulations in Castilla-La Mancha, the main features of these entities (function, composition, way of operating, etc.) are described. We conclude with a brief description of the Albacete HEC after a little over one year since its creation.

Key words. Ethics Committees, Clinical.

\section{¿PARA QUÉ SURGEN LOS COMITÉS?}

En el ámbito de la relación clínica los profesionales se enfrentan a veces a problemas cuya solución es moralmente difícil o en todo caso controvertido, problemas que pueden derivar del pronóstico del paciente, de sus deseos, de las posibilidades terapéuticas existentes o de la escasez de recursos sanitarios. En ocasiones, por ejemplo, pueden surgir discrepancias entre los médicos y los familiares en relación con el tipo de información que hay que dar al paciente en unas circunstancias muy particulares; o en relación con el esfuerzo terapéutico que resulta adecuado en pacientes sin ninguna posibilidad de recuperación, con una pésima o nula calidad de vida y mantenidos con medidas de soporte vital. O pueden enfrentarse los médicos y el propio paciente a propósito de si hay que informar de su enfermedad a terceras personas cuya salud pueda estar en riesgo debido a su relación con el paciente; o a propósito del tipo de tratamiento que éste demanda en atención a sus particulares creencias religiosas. O puede no resultar claro si debe hacerse una excepción a un protocolo clínico en atención a las muy especiales circunstancias que plantea un caso, etc. Se trata en definitiva de problemas para los que no es incuestionable cuál sea la mejor decisión a adoptar y a los que, por consiguiente, personas igualmente razonables podrían ofrecer respuestas diferentes.

Los Comités Asistenciales de Ética (CAE) nacen en los Estados Unidos en la década de los sesenta precisamente con el objetivo de ayudar a decidir en este tipo de situaciones, y desde entonces hasta ahora su número no ha hecho más que crecer, allí y en el resto del mundo. 


\section{SU PRESENCIA EN ESPAÑA}

El primer CAE de España se crea en el Hospital San Joan de Deu de Cataluña en el año 1974 y hasta hace poco tiempo la presencia de estos comités en nuestro país ha sido más bien escasa. En los últimos tiempos, sin embargo, la situación ha cambiado considerablemente, lo que sin duda obedece -entre otros factores- a que la presencia de la formación en Bioética se ha hecho mucho más intensa y a que las Comunidades Autónomas han impulsado la creación de CAE en sus respectivos territorios. Por un lado, en efecto, son los propios documentos internacionales sobre bioética que se han ido creando desde finales de los noventa hasta ahora los que han insistido especialmente en la necesidad de impulsar y expandir entre la población la formación en bioética. Así lo hacen, por ejemplo, La Declaración de Bioética del Consejo de Europa (el conocido como Convenio de Oviedo, que entró en vigor en España en 1997) y la reciente Declaración Universal sobre Bioética y Derechos Humanos de la UNESCO de 2005. Pero por otro lado, y paralelamente a este impulso hacia una mayor sensibilización de la población hacia los problemas de la bioética, las Comunidades Autónomas también han impulsado por su parte la puesta en marcha de Comités de ética asistencial allí donde sea posible por existir personal con formación en bioética. En particular, la Junta de Comunidades de Castilla-la Mancha ha regulado la constitución y funcionamiento de los Comités Asistenciales de Ética en su territorio por medio del Decreto 95/2006, de 17 de julio. Sea como fuere, lo cierto es que hoy en la mayoría de los grandes hospitales públicos y de las áreas de atención primaria existe un Comité de Ética Asistencial. De hecho, en Castilla-la Mancha existen cinco comités, cuatro correspondientes a las Áreas de Salud de Albacete, Cuenca, Guadalajara, Talavera de la Reina y uno correspondiente al Complejo Hospitalario de Toledo.

\section{¿QUÉ SON?}

Desde su nacimiento hasta ahora el número de funciones de los CAE se ha ampliado y hoy se considera que además de su función principal -que como se ha dicho consiste en asesorar sobre el modo de actuar en los conflictos éticos que puedan surgir en la práctica clínica- también es tarea de estos Comités impulsar la formación bioética de los profesionales de la salud, así como elaborar pautas y criterios para guiar la conducta de los profesionales en las situaciones conflictivas más relevantes o frecuentes. En los últimos tiempos, además, los Comités de Ética Asistencial están sufriendo una transformación paulatina derivando hacia órganos de asesoramiento y control no ya de la práctica clínica, sino de la organización sanitaria en su conjunto, con la misión de mejorar la calidad de todo el proceso del servicio asistencial. En particular, y en virtud del artículo 6 del Decreto 95/2006, son funciones de los CAE constituidos en Castilla-la Mancha:

a)Analizar y emitir informes que faciliten el proceso de toma de decisiones en las situaciones en que se planteen dilemas éticos.

b)Proponer protocolos de actuación para afrontar las situaciones en que se susciten dilemas éticos frecuentes.

c)Colaborar en la mejora de la calidad de la información y documentación clínica.

d) Velar por que se respete la dignidad de las personas que intervienen en la relación asistencial.

e)Promover actividades formativas en bioética para los profesionales de la Atención Primaria y Especializada.

De todos modos, y pese a estas otras funciones que hoy tienen también atribuidas, los CAE son básicamente órganos colegiados consultivos cuya principal misión consiste en emitir un informe que oriente a quien lo solicita (médicos, personal de enfermería, pacientes u órganos directivos de los servicios y centros comprendidos en su ámbito de actuación) sobre qué responder o cómo actuar ante una situación éticamente conflictiva para la que no existe (o no existe a juicio de quien plantea la consulta) una respuesta clara. Precisamente por eso el sentido y la justificación de los CAE reside en que éstos puedan ser órganos de reflexión y debate que actúen guiados sólo por la tolerancia y las buenas razones, es decir en que adopten sus decisiones sobre los problemas éticos planteados a través de un debate en el que cada miembro del Comité pueda exponer su punto de vista y haya de estar dispuesto a modificarlo a la luz de los (eventualmente mejores) puntos de vista ajenos.

Por lo demás, es importante subrayar también que los CAE se deslegitiman si en su actuación rebasan las funciones que tienen atribuidas para inmiscuirse en lo que podríamos denominar su "coto vedado". En particular, y por lo que respecta de nuevo a los CAE de Castilla-la Mancha, en ningún caso serán funciones de los Comités, en virtud del artículo 6. 3 del Decreto 95/2006:

a)Sustituir la decisión o la responsabilidad de quien ha solicitado asesoramiento.

b)Imponer decisiones.

c)Peritar o manifestarse sobre quejas, denuncias o reclamaciones en vía administrativa o judicial.

d)Emitir juicios sobre la ética de los profesionales, pacientes o usuarios implicados en los asuntos que se les sometan. 
e)Promover actuaciones jurídicas o proponer sanciones.

\section{¿QUIÉNES LOS INTEGRAN?}

Para poder alcanzar sus objetivos, los CAE deben ser grupos de personas plurales y multidisciplinares que se renuevan parcialmente cada cuatro años y que además prestan su asistencia de forma totalmente gratuita y desinteresada. Lo primero (pluralidad) significa que debe buscarse una composición que refleje el pluralismo ideológico presente en la sociedad, garantizando así que ningún punto de vista relevante quede excluido del debate. Lo segundo (multidisciplinariedad) significa que deben formar parte del Comité profesionales sanitarios de los diferentes sectores y servicios asistenciales (médicos y enfermeros, y, dentro de los primeros, internistas, intensivistas, médicos de familia, pediatras, etc.), así como personas de otros ámbitos de la sociedad, de los cuáles suele exigirse que al menos uno sea jurista con conocimientos en legislación sanitaria. En todo caso la mayoría de las regulaciones sugieren que en la elección de los miembros del CAE se considere su formación en bioética: el mencionado Decreto 95/2006, regulador de los Comités en Castilla-la Mancha, establece que en la composición de los mismos debe haber al menos "una persona de reconocido prestigio, ajena al ámbito sanitario y preferentemente con formación acreditada en ética" (artículo 3.1. d); y la misma norma, más adelante, reitera que "al menos uno de los miembros de cada Comité habrá de ser una persona con formación superior en bioética" (artículo 3.5).

\section{¿CÓMO ACTÚAN?}

En el desempeño de su principal tarea, que es el análisis y asesoramiento de los conflictos éticos que se suscitan en la relación asistencial, los CAE actúan de dos modos: autónomamente y a demanda. En el primer supuesto son los propios Comités los que espontáneamente elaboran guías o protocolos que puedan orientar la actuación de los profesionales (y de los pacientes) en los conflictos más frecuentes y/o que se atisba pueden ser una fuente de problemas en un futuro inmediato (por ejemplo, el Comité puede establecer unas líneas generales de actuación que orienten a los facultativos sobre qué hacer ante el rechazo de los Testigos de Jehová a los tratamientos con hemoderivados).

Pero lo normal es que el Comité intervenga a demanda, bien de los pacientes y usuarios de la sanidad o de los órganos directivos de los correspondientes centros y servicios, bien -como suele ser lo habitual- de los profesionales sanitarios, que preguntan cómo deben actuar en un caso clínico concreto en que esto no está claro. Cuando en el seno del CAE se analiza el caso se examina en primer lugar si el Comité es competente para responder a lo que se pregunta; y después, si lo es, se identifican todos los problemas éticos suscitados, se introducen en el análisis todos los valores implicados y se evalúan todos los cursos de acción posibles antes de justificar y proponer alguno o algunos de ellos. Por lo general en los Comités no se emiten votos, sino que se debaten los asuntos a tratar y normalmente se alcanza un acuerdo o unas conclusiones; lo que no quita para que en casos especialmente conflictivos donde el consenso total no es posible pueda y deba votarse.

En cualquier caso es importante insistir en que los CAE son órganos consultivos y que por lo tanto sus informes no son vinculantes sino que tienen un carácter meramente asesor. Esto significa que el profesional que ha elevado la consulta puede hacer caso omiso de la orientación contenida en el informe. Pero significa también que, si decide ajustar su actuación a lo sugerido o recomendado en el informe, ello no le exime de su eventual responsabilidad profesional y personal. La opinión contenida en el informe tan sólo pretende ofrecer ayuda para poder adoptar una decisión con fundamentos éticos.

Por último, y como resulta obligado -entre otras- por la normativa vigente sobre protección de datos, los Comités actúan bajo el deber de confidencialidad y de secreto, deber que se proyecta sobre toda la información a la que los miembros del Comité tengan acceso en el cumplimiento de sus funciones.

\section{EL CAE DE ALBACETE: A MODO DE BALANCE}

El Comité Asistencial de Ética del Área de Salud de Albacete se constituyó por Resolución de la Dirección-Gerencia del SESCAM de 29 de octubre de 2008. Cuenta con 15 miembros, de los cuales trece son profesionales sanitarios (un intensivista, una inspectora médica, una rehabilitadora, un médico de urgencias, dos de atención primaria, un pediatra, un urólogo, una paliativista, tres enfermeros y un trabajador social) y dos son juristas.

Desde que se constituyera hasta ahora ha seguido ampliándose la formación en bioética de los miembros del Comité pero, sobre todo, se ha consolidado su funcionamiento. EI CAE se reúne en sesiones ordinarias una vez al mes y extraordinarias cuando se plantea una consulta que requiera rapidez en la respuesta.

Durante 2009 el CAE ha desarrollado la siguiente actividad:

$\left.1^{\circ}\right)$ Ha emprendido autónomamente un estudio sobre La atención sanitaria a los Testigos de Jehová, con el objetivo último de articular, si fuera posible, una pauta de actuación clínica ante los pacientes que profesan esta creencia religiosa.

$\left.2^{\circ}\right)$ Se han analizado e informado tres casos clí- 
nicos, dos de los cuales han sido planteados por facultativos y el tercero por una paciente. $Y$ por lo que se refiere al sentido de los informes emitidos, en dos casos se ha hecho una recomendación de actuación y en el otro se ha resuelto la falta de competencia del Comité.

$\left.3^{\circ}\right)$ Por último, se ha solicitado asimismo al CAE pronunciamiento sobre dos protocolos de actuación, pero también en estos casos se ha resuelto la falta del competencia del Comité.

En relación con estas resoluciones de "falta de competencia" merece la pena destacar dos cosas. La primera es que el Comité del Área de Salud de Albacete ha querido ser sumamente escrupuloso con el deslinde de sus funciones, a fin de no aconsejar y ni siquiera sugerir cursos de acción ante cuestiones que no son competencia del Comité sino que pertenecen en exclusiva al criterio clínico. La segunda, y directamente relacionada con la anterior, es que la mayor parte de estas resoluciones provienen a nuestro juicio de que todavía no existe, entre los profesionales y/o los pacientes que plantean las consultas, una idea cabal del sentido y las funciones de un CAE, por lo que tal vez deberían hacerse esfuerzos en este sentido. Precisamente en consonancia con este esfuerzo pendiente el CAE de Albacete está preparando la realización de pequeñas sesiones destinadas a difundir la formación en bioética entre profesionales sanitarios y no sanitarios.

\section{¿CÓMO DIRIGIRSE AL CAE DEL ÁREA DE SA- LUD DE ALBACETE?}

\section{¿Quién y cómo puede solicitar asesoramiento?}

Cualquier profesional sanitario, o cualquier paciente o usuario, o cualquier directivo de un Centro o establecimiento sanitario del SESCAM puede soli- citar asesoramiento al CAE mediante un simple escrito en el que exponga con claridad qué problema origina su consulta y qué se pregunta.

¿A quién y a dónde enviar la solicitud?

El escrito ha de dirigirse al Presidente del Comité de Ética Asistencial, bien por correo ordinario (Hospital General de Albacete, C/ Hermanos Falcó, s/n, 02006-Albacete) o bien por mail (cetica@sescam. jccm.es).

\section{BIBLOGRAFIA ORIENTATIVA}

- Martínez JL. Comités de Bioética. Universidad Pontificia de Comillas; 2003.

- Ribas, S. Los comités de ética asistencial en Estados Unidos. Healthcare Ethics Comittes in U.S. Madrid: Institut Borja di Bioètica-Fundación MAPFRE; 2003.

- Simón Lorda P. Ética de las organizaciones sanitarias. Nuevos modelos de calidad. Madrid: Triacastela; 2005.

\section{NORMATIVA DE INTERES}

- Declaración Universal sobre Bioética y Derechos Humanos de la UNESCO, de 19 de octubre de 2005.

- Convenio para la Protección de los Derechos Humanos y la Dignidad del ser humano con respecto a las aplicaciones de la Biología y la Medicina, hecho en Oviedo el 4 de abril de 1997 (Convenio de Oviedo).

- Ley 41/2002, de Autonomía del paciente y de derechos y obligaciones en materia de información y documentación clínica.

- Decreto 95/2006, de 17 de julio, de la Consejería de Sanidad, de los Comités de Ética Asistencial en el ámbito del SESCAM.

- Circular 6/2006, de 3 de octubre, de la Dirección-Gerencia, sobre Constitución y Funcionamiento de los Comités de Ética Asistencial. 